\title{
Une vie dédiée à Clio sous le prisme de la loi, du Hainaut et des ducs de Bourgogne. Interview de Jean-Marie Cauchies
}

Nicolas Simon \& Quentin Verreycken

Nicolas Simon : Diplômé en histoire de l’UCL (2011), Nicolas Simon a également réalisé un master complémentaire en sociologie-anthropologie (UCL, 2012). Il est actuellement Aspirant F.R.S-FNRS. L'axe principal de ses recherches s'insère dans les champs de l'histoire du droit, en particulier celle de la législation, et de l'histoire interne des institutions. Sa thèse de doctorat porte sur l'étude du processus décisionnel au niveau législatif dans les Pays-Bas espagnols (fin XVIe-début XVIIe siècle).

Quentin Verreycken : Diplômé en histoire de l’Université catholique de Louvain (2013), Quentin Verreycken est depuis octobre 2014 aspirant FRS-FNRS en co-tutelle UCL-Université Saint-Louis. Il est également diplômé de la KU Leuven (Master of Medieval and Renaissance Studies, 2014). Ses recherches s'articulent, d'une part, autour de l'histoire de la violence et de son contrôle au travers de la pratique de la rémission princière à la fin du moyen âge, et d'autre part autour de l'évolution des structures militaires sous les ducs de Bourgogne. La thèse qu'il prépare portera sur le droit de grâce et la construction étatique en Angleterre, France et anciens Pays-Bas au XVe siècle.

DOI: $10.25518 / 1370-2262.346$

\section{Introduction}

Dans le paysage de la recherche historique belge, le professeur Jean-Marie Cauchies occupe une place singulière. Il est surtout et avant tout un historien intéressé par l'histoire de la loi, du fait législatif comme il aime à le dire. J.-M. Cauchies se frotta assez vite à l'étude de la législation au cours de ses études universitaires. C'est au moment de choisir un sujet pour son mémoire de licence (actuel master) que cette thématique de recherche lui fut conseillée par Maurice Arnould, professeur d'histoire des temps modernes à l'Université libre de Bruxelles, à l'identité hainuyère, tout comme J.-M. Cauchies. Articulant son intérêt pour l'histoire de la loi avec celle pour ses terres d'origine, le Hainaut, et sa période de prédilection, le $15^{\mathrm{e}}$ siècle bourguignon, J.-M. Cauchies n’a cessé, depuis quarante ans, de labourer un terrain fécond et d'offrir à la communauté des chercheurs de nombreuses publications souvent précieuses pour notre compréhension du processus législatif à la fin du moyen âge. Depuis la rentrée de l’année académique 2012-2013, J.-M. Cauchies est professeur émérite invité à l’Université Saint-Louis - Bruxelles.

Posons peut-être la question que d'aucuns se poseraient: pourquoi avoir choisi de suivre une formation en histoire aux Facultés universitaires Saint-Louis (actuelle Université Saint-Louis Bruxelles) ? Pourquoi avoir décidé de mener des recherches à propos de l'histoire de la législation à l’Université catholique de Louvain ? À la première question, Jean-Marie Cauchies indique volontiers que sa réponse est « très banale » puisqu'il s'intéressa à l'histoire dès l'école primaire. De son propre aveu, il s'est rapidement «senti attiré par le passé ». Il dit encore : « Ça n’a pas été un chemin de Damas; c'est quelque chose qui s'est construit petit à petit ». L'attrait pour la 
Une vie dédiée à Clio sous le prisme de la loi, du Hainaut et des ducs de Bou...

législation vint quelques années plus tard, au moment d'entamer ses années de licence en histoire à l'Université catholique de Louvain. Si l'étude des ordonnances du duc Philippe le Bon pour le comté de Hainaut ne le rebutait pas, c'est sans doute en raison de son caractère. J.-M. Cauchies dit lui-même ceci : «Je suis un homme d'ordre, un homme de normes ; j'aime bien ce qui est défini, ce qui est cadré. Je crois qu'il ne faut pas poursuivre plus loin l'introspection » si l'on veut comprendre pourquoi J.-M. Cauchies a voué sa carrière scientifique au fait législatif. L'importance du troisième membre du trio indiqué dans le titre - la période bourguignonne - est à mettre au crédit d'un petit livre consacré à Philippe le Bon justement et qui avait été offert à J.-M. Cauchies au cours de son enfance. «Je pense que c'est ceci qui a produit le déclic vers l'époque bourguignonne » dit l'intéressé. Des Bourguignons aux premiers Habsbourg (Maximilien d'Autriche notamment), il n'y a qu'un pas que J.-M. Cauchies a franchi allègrement dès sa thèse de doctorat (soutenue en 1978 et publiée en 19821). Au moment où il entame d'ailleurs sa thèse - nous sommes en 1972 - la "période habsbourgeoise était très peu étudiée, mais la situation a bien changé grâce notamment aux historiens gantois ». Travaillant et re-travaillant cette période comme peu avant lui l'avaient fait, il en est progressivement venu « à considérer que cela formait un tout » avec le prétendu âge d'or bourguignon.

Après avoir terminé ses études d'histoire en 1972 - il n’a alors que 21 ans - il entama une thèse de doctorat à l'Université catholique de Louvain $\underline{2}$ sous la direction de Philippe Godding $\underline{3}$. Il obtint d'ailleurs un mandat d'aspirant auprès du Fonds national de la recherche scientifique (FNRS) pour réaliser cette entreprise. Il gravit ensuite sans encombre les échelons du parcours académique, passant de suppléant (1977) à professeur ordinaire (1992) aux Facultés universitaires Saint-Louis. En parallèle, il fut professeur (à temps partiel) à l'Université catholique de Louvain où il dispensa notamment le cours d'histoire de Belgique qu'il donnait déjà aux étudiants de son Alma Mater bruxelloise.

Durant sa carrière, J.-M. Cauchies fut et est encore un chercheur aux multiples attaches. Non seulement il participa à la création d'Hannonia, structure fédérant les cercles d'histoire locale en province de Hainaut belge, mais fut également pendant plus de trente ans le secrétaire exécutif du Centre européen d'études bourguignonnes (XIV $-\mathrm{XVI}^{\mathrm{e}}$ s.). À la figure de l'historien et de l'académique s'adjoint donc celle de l'homme de réseaux. Cette reconnaissance par les pairs se manifeste ainsi par une présence dans diverses institutions et commissions scientifiques : Académie royale de Belgique (depuis 20024), Commission royale d'histoire (depuis 1996) ou la Commission royale pour la publication des anciennes lois et ordonnances de Belgique (depuis 1982). Il est également le co-fondateur du Centre d'histoire du droit et des institutions (CRHiDI) dont il a été directeur de 1992 à 2012.

On découvrira au cours de cette interview une pensée riche et ouverte, tant sur l'historiographie française, indispensable pour ce spécialiste des ducs de Bourgogne, qu'allemande, où la réputation des recherches en histoire du droit n'est plus à démontrer. J.-M. Cauchies cite volontiers les travaux d'Herman Wiesflecker concernant Maximilien d'Autriche, les biographies de Richard Vaughan consacrés aux ducs de Bourgogne comme des ouvrages majeurs qui l'ont influencé. En revanche, on ne trouvera pas chez J.-M. Cauchies des références à des penseurs polymorphes situés aux frontières de plusieurs disciplines, tels des Michel Foucault ou des Max Weber. Le principal intéressé s'en explique d'ailleurs sans faux détour. "Plus globalement, j'appartiens à une génération et j'ai été formé par des gens qui, sans rejeter ce genre d'auteurs, étaient relativement méfiants. (...) En conclusion, je ne suis pas tombé là-dedans comme Obélix dans la cuvelle de potion 
magique quand j'étais petit. Je n'ai donc jamais pris ce réflexe de consulter des auteurs issus d'autres disciplines puisque je trouvais ma nourriture chez les historiens, même si j'allais chercher quelques informations spécifiques chez des juristes contemporains ».

L'interview qu'on lira ci-dessous est une partie d'un entretien plus large que nous avait accordé J.-M. Cauchies au printemps 2015. Dans le cadre du présent numéro des C@hiers du CRHiDI,nous avons choisi de ne reprendre ici que les échanges concernant les travaux de J.-M. Cauchies relatifs à l'histoire du fait législatif.

\section{Interview}

Nicolas Simon \& Quentin Verreycken (NSQV) - Dans le titre d'un de vos articles consacrés à l'histoire de la loi $\underline{5}$, vous vous demandiez si l'histoire de la législation n'était pas un champ de mines (mijnenveld) ? Quelles sont les mines qui parsèment l'étude de la loi ?

Jean-Marie Cauchies (JMC) - Il faut peut-être rappeler le contexte dans lequel cet article est né. Au départ il s'agissait d'une conférence que j'ai prononcée, en français, lors de l'éméritat de Guido Van Dievoet (KU Leuven). Pour la séance officielle au cours de laquelle on lui remettait également ses Mélanges, il fallait un orateur. Jos Monballyu et Denis Vanden Auweele - les deux professeurs d'histoire du droit - m'avaient contacté. J'ai ainsi choisi d'aborder l'histoire de la législation via l'aspect méthodologique tout en trouvant un titre accrocheur. J'ai ensuite eu l'occasion de le publier, en néerlandais, dans les actes du colloque belgo-néerlandais d’histoire du droit.

\section{NSQV - La référence aux mines n'était qu' « esthétique » pour le titre ?}

JMC - Non, ce n'est pas qu'un titre. Parler d'histoire de la loi ou de la législation ne convainc pas tout le monde. Du moins pour les périodes envisagées (moyen âge et début des temps modernes), on fait valoir que les termes n'existent pas. Pour certains juristes et même historiens du droit, c'est extrêmement redoutable. Ce serait donc la première mine sur laquelle il ne faut pas sauter. La seconde mine renvoie au travers dans lequel sont tombés les historiens du droit du $19^{\mathrm{e}}$ siècle - ce qui n'enlève évidemment rien à leurs mérites, mais ils étaient davantage des juristes et magistrats que des historiens - à savoir transposer des réalités actuelles aux périodes médiévale ou moderne. Fidèle au chiffre ternaire, je relèverais une troisième mine, même s'il y en a encore d'autres. Il s'agit de la question des auteurs. Par qui est produite une loi ? Par quoi ? Qui dit législation dit aussi législateur. Mais qui est réellement le législateur ? Comment nait une loi ? Voilà pour les mines et je dirais que je n’ai pas encore explosé...

NSQV - Rebondissons, sans sauter, sur l'une des mines que vous avez relevées. Envisager l'étude de la législation au moyen âge ne va pas de soi, ou du moins n'a pas toujours été de soi. Pouvez-vous nous expliquer pourquoi certains historiens ont pu avoir une réticence à parler de « législation » ou de « pouvoir législatif » pour le moyen âge ?

JMC - Nul ne contestera, me semble-t-il, parmi les historiens qui étudient la législation que le fait législatif existe. Il y a des normes qui règlent. Je crois, en effet, que le débat porte sur la façon de nommer ce fait législatif. La réponse m’apparaît très simple et cela m’a vraiment frappé au fil des années. Il existe une distorsion complète entre les historiens français et allemands. Les collègues allemands n'hésitent pas à parler de Gesetz, Gesetzgebung, etc. Du côté français, les plus expérimentés (Albert Rigaudière, Jacques Krynen, Yvonne Bongert) utilisent l'expression 
Une vie dédiée à Clio sous le prisme de la loi, du Hainaut et des ducs de Bou...

de «pouvoir normatif». Je ne pense pas que ce soit une divergence, mais davantage une question d'oser s'engager dans les termes. J'ai eu l'occasion d'en discuter directement avec Albert Rigaudière. Je ne dirais pas que j'ai réussi à le convaincre entièrement, mais il paraissait plus à l'aise qu'un autre chercheur français à utiliser le mot «législation ». Néanmoins quand il utilise l'expression « pouvoir législatif » dans un article il faut qu'il y ait une glose sinon un commentaire pour justifier cet emploi. Un auteur allemand comme Friedrich Ebel, qui a écrit une petite histoire de la loi en Allemagne, ne se croit pas obligé d'expliquer pendant deux pages le recours aux mots Gesetz ou Gesetzgebung‥ En France, on est beaucoup plus prudent. Je pense, au final, qu'il s'agit d'une question de culture. Ce n'est certainement pas un débat sur le fond visant à dire qu'il n’y a pas quelque chose qui correspond à notre législation. Pourquoi cette différence ? Je pense que les Français, sans en être toujours pleinement conscients peut-être, sont irréductiblement marqués par leur jacobinisme. Il y a des termes (l’État, le législateur, la loi, etc.) qu'ils n'utilisent qu'à partir du moment où on a une autorité qui est bien orientée. En gros, les historiens français sont tous un peu les enfants de la Révolution française tandis que la tradition allemande, beaucoup plus parcelliséę, n’hésite pas à reconnaître que certains princes territoriaux disposent d'une puissance législative.

\section{NSQV - Vous n'avez donc aucun problème à recourir à l'expression « pouvoir législatif »?}

JMC - Les historiens français ne m’ont jamais voué aux gémonies parce que je parlais de législation. Mais je pense qu'ils comprennent aussi que bien qu'utilisant les mêmes mots qu'eux, je viens d'une culture nourrie autrement. Je peux déjà dire que dans mon projet de synthèse d'histoire de la législation, j'aurai plaisir à consacrer plusieurs pages à ce débatㅁ․ Il est évident que " pouvoir normatif » inclut la loi tandis que le pouvoir législatif n’est pas tout le normatif.

\section{NSQV - Est-ce que ce qui vous intéresse dans l’étude de la législation est toujours de « gratter le vernis des prescriptions pour mieux dégager le bois des réalités quotidiennes " $\underline{9}$ ? Pourquoi pareille approche ? Comment procéder?}

JMC - Dès le départ j’ai été mis sur la bonne voie, si on peut dire. Je garde une reconnaissance éternelle envers Maurice Arnould10. Étudier les textes législatifs pour eux-mêmes ne l'intéressait pas vraiment car il n'était pas spécifiquement historien du droit. Il avait commencé par la démographie historique et était davantage historien de la société que des institutions. Il m’a alors dit qu'en travaillant sur les dénombrements de feux11 il avait trouvé des mentions à propos des textes législatifs. C'est donc lui qui m’a mis en piste là-dessus. Quand je suis arrivé à Louvain pour la licence et que j'ai évoqué ce que m’avait proposé Maurice Arnould, Léopold Genicot a dit (il imite le phrasé de Genicot) : "Ah oui, il y a moyen de faire beaucoup de choses à partir des comptes ». Inévitablement, je n'ai pas abordé l'étude de la législation comme l'eut fait un historien du droit d'autrefois, en rassemblant ses textes et analysant la façon dont ils ont évolué. En parallèle avec l'établissement d'une liste chronologique, j'ai donc touché la matière pratique. C'est cela le « bois des réalités quotidiennes ». Dans le fonds de la Chambre des comptes à Lille, par exemple, il y a cette section de la correspondance dite des lettres reçues et dépêchées qui ne paraît pas fournir beaucoup d'informations si on n'a pas ses repères, alors qu'elle permet d'enrichir les dossiers. On se rend compte du fonctionnement des institutions et on en vient à pouvoir étudier les travaux préparatoires à la législation.

NSQV - Vous avez d'ailleurs un peu alterné les types d'approches. 
JMC - Oui. Aux rencontres du Centre européen d'études bourguignonnes à Dijon (1980), j'avais présenté un panorama sur les premiers essais d'une législation générale au $15^{\mathrm{e}}$ siècle. C'était la vision des textes. Ensuite, aux rencontres de Rotterdam (1983), je me suis référé à la communication de Dijon en disant que j'avais pris les choses « par le haut » et que je comptais maintenant aborder la question «par le bas ». J'ai alors commencé à analyser des documents d'approche. Je n'ai donc pas dû faire d'efforts pour rentrer dans la démarche. Mes bons maîtres, tant Arnould que Genicot puis surtout Godding, mon promoteur, m'ont tout de suite mis sur la route.

NSQV - Dans ce même article vous lanciez un avertissement : un texte législatif ne s'improvise pas et pour bien comprendre une décision il ne faut pas s'en tenir au produit fini. C'est un leitmotiv qui traverse tous vos travaux. Pourquoi est-ce important d'insister là-dessus ?

JMC - Là, il s'agit de gratter un petit peu plus loin que ce qu'on va trouver dans les comptes. On en vient à se poser cette question : pourquoi une décision là-dessus et à tel moment ? Si on consulte les éditions de sources, c'est un « enregistrement » dans la mesure où on voit que telle année il y a une ordonnance sur telle matière, etc. On peut évidemment tirer des conclusions sur cette première base. Il ne faut pas s'étonner qu'au début d'un règne il y ait soit une série de renouvellements de privilèges ou de nouveaux qui sont accordés. C'est très facile à comprendre. Par contre on peut parfois rencontrer un texte inattendu, un hapax à propos duquel on peut s'interroger. Il faut alors essayer d'aller au-delà de la simple lettre de l'acte et voir ce qui a motivé la promulgation.

\section{NSQV - Vous auriez un exemple en tête?}

JMC - Au moment où le futur Louis XI $\left({ }^{\circ} 1423-^{\dagger}{ }^{14483)}\right.$ exilé séjourne au château de Genappe, en Brabant, il y a une ordonnance sur la chasse qui est promulguée. Ce n'est pas courant de voir une ordonnance sur cette thématique. Qu'apprend-on en recoupant les sources ? Le dauphin allait beaucoup chasser durant son séjour12. L’ordonnance a vraisemblablement bénéficié de l'implication du futur roi de France car on y trouve une allusion dans des sources connexes. Devant un cas pareil, on se dit qu'il est important d'essayer de connaître les facteurs parfois ténus qui ont pu conditionner les choses. Néanmoins, pouvoir souligner que le produit fini ne suffit pas implique une bonne connaissance de l'environnement qu'on étudie. Ce n'est qu'en travaillant de manière systématique sur une époque et un territoire qu'on peut comprendre ceci. Avec un texte ou deux, on ne peut pas le faire.

\section{NSQV - Au fond, on peut dégager plusieurs « stades » dans l'approche de la législation et de son élaboration.}

JMC - Je dégagerais trois stades. Il y a celui du rassemblement des textes, la mise en série. Un deuxième stade - qui peut précéder l'autre - serait le dépouillement de la documentation comptable ou de la correspondance qui permet de repérer des mentions de textes qu'on a conservés ou non. Le troisième stade invite à poursuivre l'introspection encore plus loin, aller au-delà du dit pour atteindre le non-dit et déterminer pourquoi un texte survient à tel moment et pas un autre. C'est ce que j'ai fait pour une contribution à un colloque organisé début 2015 à Genève sur les Statuta Sabaudiae13. Je me suis demandé ce qui à l'époque pouvait motiver ou non la rédaction de certains textes. J'ai pu retrouver des choses dont je n'avais pas eu conscience nécessairement avant : pourquoi telle disposition apparaît-elle ici et ailleurs au même moment ? Je me suis rendu compte que dans les Pays-Bas bourguignons où, a contrario de la Savoie, on en est encore au stade 
Une vie dédiée à Clio sous le prisme de la loi, du Hainaut et des ducs de Bou...

des balbutiements institutionnels, il y a une mobilité des hommes. Comme si les institutions se les «prêtaient ». Je ne dis pas que c'était inconnu des historiens, mais ça m'est beaucoup mieux apparu avec certains membres du personnel de la Chambre des comptes de Lille que l'on peut retrouver en Hollande. C'est la confrontation de textes normatifs avec ce que l'on sait par ailleurs des carrières de tel ou tel membre que l'on peut se rendre compte qu'il y a un « pullulement ».

NSQV - Arlette Jouanna met en avant que « Légiférer n’était nullement considéré comme un devoir impératif des gouvernants; le besoin ne s'en imposait que pour remédier à des abus ou combler les lacunes. D'où le caractère de 'bouche-trous' que revêtaient les ordonnances " 14 . On remarque qu'au $16^{\mathbf{e}}$ siècle, dans les Pays-Bas espagnols, nombre d'ordonnances reprennent des dispositions mentionnées dans des actes antérieurs et que les avis de conseils provinciaux préconisent en premier lieu de republier d'anciennes ordonnances plutôt que de promulguer de nouvelles dispositions. Vous avez vous-même parlé de «filiation» entre les actes. N'est-ce pas le signe d'un rapport tout à fait particulier au passé, mais aussi au pouvoir du prince ?

JMC - Ce rôle du passé est très déterminant. Quand il y a des références à des choses anciennes qui ne sont pas nécessairement précisées, jadis on avait peut-être tendance à considérer qu'il s'agissait de formules générales sans beaucoup d’importance. Je pense au contraire que derrière une référence il y a toujours une réalité qui se profile. Parfois elle est explicite, comme lorsqu'il est écrit « L'ordonnance de feu mon père de telle année... ». Dans ce cas-là, c'est clair. Dans un article ancien j'avais analysé les serments prêtés par les comtes de Hainaut dans lesquels on mentionne de façon assez vague d'autres textes 15. Les auteurs de jadis disaient qu'on renvoyait sans discernement aux « anciennes chartes ». Je crois avoir pu établir, preuves à l'appui, qu'il s'agissait de choses bien précises. Donc une relation à un passé qui n'est pas flou ou de pure référence, mais qui correspond vraiment à des réalités. Pour la charte générale de Hainaut de Maximilien d'Autriche et de Philippe le Beau (1483), par exemple, on fait ainsi allusion à des textes antérieurs. Quand on connaît les « rétroactes », je crois qu'on peut préciser à quels textes on fait allusion. Il y a un incontestable souci de filiation et beaucoup plus de précision dans l'esprit des rédacteurs que ce que l'on imagine.

\section{NSQV - Et concernant les propos d'Arlette Jouanna?}

JMC - Je suis d'accord avec cette formule. Je vais encore me référer à Genicot. Il disait à propos des chartes de franchises - pour lesquelles on dispose moins de documentation connexe - qu'on ne doit pas s'imaginer qu'à l'époque on mettait tout par écrit. Si l'on mettait quelque chose par écrit, c'est qu'on éprouvait la nécessité de le faire. L'expression «bouche-trous » est peut-être un peu provocatrice, mais elle n'est pas inexacte. Quand on fait un texte c'est que visiblement il y a des choses qui ne sont pas réglées ou qui ne fonctionnent plus. On ne fait donc pas un texte pour la mémoire. On le fait parce qu'on en éprouve la nécessité. Pour reprendre Genicot, l'écrit est quelque chose d'exceptionnel, on met par écrit ce qui est a-normal, ce qui s'écarte de la norme vécue.

NSQV - Depuis quelques années justement, on observe le développement d'un mouvement historiographique lié aux pratiques de l'écrit via les travaux de l'anthropologue Jack Goody, qui ont influencé les historiens. Pour vous, ce sont des approches qui vont de soi ou bien cela enrichit le questionnaire de l'historien?

JMC - Cela me fait penser à une remarque de Ludo Milis $\underline{16}$ lors de la soutenance publique de 
la thèse défendue par Jacques Nazet à l'Université libre de Bruxelles, en 198917. La question avait complètement déstabilisé J. Nazet et étonné l'assistance. L. Milis a ainsi dit à Nazet : « Vous expliquez ce que ces chanoines écrivaient, mais pourquoi écrivaient-ils ? ». Ce fut un dialogue de sourd. De manière assez positiviste j'aurais répondu comme Nazet qui disait que les moines écrivaient parce qu'ils avaient des choses à régler. Milis voulait quant à lui savoir pourquoi ils pratiquaient l'acte d'écrire. La question paraissait alors d'avant-garde, ce qui a surpris le récipiendaire et a dû en surprendre bien d'autres. Dès lors, c'est poser de bonnes questions que de s'intéresser à cela aujourd'hui. D'ailleurs, se demander pour quoi un acte législatif est écrit est encore facilement explicable. Se demander pourquoi on écrit, c'est autre chose.

\section{NSQV - Vous ne vous êtes jamais posé ces questions?}

JMC - À vrai dire je ne me suis jamais posé ces questions consciemment, mais dans l'optique notamment des travaux de Paul Bertrand18, cela vaut le coup de se les poser. D'ailleurs, dans la thèse de Marie Van Eeckenrode, on sent bien qu'elle est marquée par cette problématique19. Il y a des passages forts intéressants et même un chapitre consacré à l'acte d'écriture au sein des États du comté de Hainaut. Je pense que c'est une bonne chose et peut-être qu'un jour je devrai moimême m'y mettre pour des textes que j'ai déjà expérimentés. Autrefois, les historiens auraient pu répondre qu'on a mis par écrit parce qu'on ne savait pas faire autrement. Pourtant il y a d'autres moyens de communication et certaines choses ont été publiées sans avoir été mises par écrit et ce à une époque où tout le monde ne sait pas lire. C'est quelque chose, par exemple, que je voudrais approfondir à propos de la législation en partant des considérations que j'ai déjà pu faire sur les bretèches $\underline{20}$.

\section{NSQV - Dans les sources d'inspiration pour de nouvelles dispositions, il est assez patent de ne quasiment jamais retrouver la mention de dispositions relevant de la « jurisprudence ». L'inverse n'est pas vrai. Pour quelle raison selon vous?}

JMC - Je répondrais de manière un peu directe et brutale. Il existe des juges et une justice, mais il n'existe pas de jurisprudence comme nous l'entendons. Dans son ouvrage, Alain Wijfels 21 met en avant que dans le cadre de leur activité les juges se réfèrent à des coutumes, des ordonnances, des allégations de droit romain, etc. Cette notion de jurisprudence est en quelque sorte inventée. Elle existe dans une certaine mesure en Angleterre parce qu'il s'agit d'une autre tradition bien qu'on ne doit sans doute pas définir le common law comme une jurisprudence. Si vous voulez, la jurisprudence n'existe pas. Il eut été incongru et peut-être même attentatoire à la souveraineté d'aller, dans un texte émanant du législateur, se référer à ce qu'a pu dire un juge. Je ne vois pas une manière de concevoir le droit qui soit favorable à cette utilisation.

NSQV - Même dans le cas de jugements émis par une cour comme le Grand Conseil de Malines au $16^{\mathbf{e}}$ siècle, qui rend la justice au nom du prince ?

JMC - Oui, mais ce n'est pas le prince. Dans un article, Hugo De Schepper s'est justement demandé quelle était la première cour de justice des anciens Pays-Bas2․ Est-ce que c'est le Grand Conseil de Malines ou le Conseil privé qui est plus proche de la personne du prince ? J'ai l'impression que cette spécificité du Conseil privé (proximité avec le prince) est à retenir. Ce genre de question ne me paraît toutefois guère envisageable pour le $15^{\mathrm{e}}$ siècle. Pour le $16^{\mathrm{e}}$ siècle, on a peut-être pu prendre en compte des décisions prises à Malines pour élaborer la législation, mais ça me semble peu concevable de les exprimer dans une ordonnance. C'est peut-être discutable comme position et 
Une vie dédiée à Clio sous le prisme de la loi, du Hainaut et des ducs de Bou...

on me dira que pour le $16^{\mathrm{e}}$ siècle elle est même brutale. Pour le $15^{\mathrm{e}}$ siècle, je serais plus affirmatif.

NSQV - Depuis la Révolution française, la loi est chez nous - pour reprendre les mots de François Ost - «l’astre solaire au cour de la nébuleuse normative »23. L'efficacité d'une assemblée législative est aujourd'hui perçue selon la quantité de lois déposées et les centaines, voire les milliers de pages que cela engendre. Pour les périodes que vous envisagez dans vos recherches la loi a-t-elle déjà cette importance ?

JMC - La loi va assurément devenir de plus en plus un instrument de gouvernance, mais il s'agit rarement de la loi à l'état pur. On trouve énormément d'éléments déjà présents dans les coutumes et parfois on n'a pas mauvaise conscience de l'exprimer. Ceci explique le phénomène de rassemblement et de mise par écrit des coutumes à travers les lois. La formule de François Ost est très belle et correspond à une réalité qui nous vient du Régime français (1795-1815). On va alors considérer qu'il faut les mêmes règles de droit pour tous et que ces règles de droit ne peuvent avoir pour source que le législateur. Aux $15^{\mathrm{e}}-16^{\mathrm{e}}$ siècles, la loi n'a pas encore cette importance même si elle commence à en prendre. On peut certes toujours dire qu'on a perdu des textes. Toutefois quand on voit - même pour des périodes antérieures au $15^{\mathrm{e}}$ siècle - la quantité de textes coutumiers, même si ce sont des bribes, ainsi que la quantité sensiblement limitée de textes que l'on peut qualifier de (para)législatifs, il est clair que la loi n'occupe pas encore tout le terrain.

\section{NSQV - C'est le côté « bouche-trou » de la loi dont parle Arlette Jouanna?}

JMC - Oui. La loi ne va pas nécessairement créer du neuf. Elle peut reprendre des éléments coutumiers qui pouvaient suffire par leur existence. Le fait que le prince les exprime par la loi est susceptible de leur donner plus de poids, mais il ne va peut-être le faire que si cela s'avère vraiment nécessaire. Bref, il y a un trou à boucher ou une filiation à mieux assumer avec des coutumes. La loi a donc un poids moins grand par rapport à cette source prédominante qu'est la coutume. Et puis il y a d'autres usages que nous ne connaissons pas parce qu'ils n'ont pas été mis par écrit et ne le seront que tardivement. Plus on se rapproche du niveau local et plus on le constate. Quand on voit les dispositions de certaines ordonnances qui ont une application très locale (entretien des cours d'eau, voirie, etc.) il faut parfois aller chercher les origines des mesures dans des textes antérieurs strictement locaux et qui n'émanent pas du prince.

NSQV - Vous avez écrit qu'avec l'arrivée des ducs de Bourgogne au $15^{\mathrm{e}}$ siècle dans les anciens Pays-Bas, une progressive hiérarchie des normes se met en place en même temps qu'une autre hiérarchie prend forme, celle des corps politiques et administratifs $\underline{24}$. Est-ce que vous pouvez expliquer quelque peu le phénomène?

JMC - C'est l'examen, en parallèle, du développement d'une législation qui se fait de plus en plus prenante territorialement avec le développement d'institutions dont le champ de compétences se fait plus large aussi. Prenons le comté de Flandre sous Philippe le Hardi et Jean sans Peur (1386-1419). Beaucoup de textes sont promulgués mais peu s'appliquent sur l'ensemble du territoire comtal. C'est aussi un moment où les institutions compétentes pour tout le comté se mettent seulement en place. Je ne vais pas dire qu'il n'existe pas d'institutions pour tout le comté vu qu'il y a déjà un Conseil et qu'une Chambre des comptes va être créée. C'est donc parallèlement à ceci qu'apparaissent des textes plus prégnants au niveau du territoire. On arrive ensuite au développement d'institutions dites centrales en même temps que se prennent des mesures que l’on peut qualifier de générales pour la facilité, mais qui ne sont peut-être pas si générales que 
cela comme certains l'ont montré avec bonheur25. Elles ne sont pas vraiment générales car si on s'efforce de les appliquer partout c'est selon des canaux qui restent spécifiquement régionaux. Cela n'apparaît peut-être pas très bien pour le $15^{\mathrm{e}}$ siècle car la documentation est encore limitée, mais le phénomène paraît plus évident au $16^{\mathrm{e}}$ siècle. Dès lors, même à une époque où des institutions centrales s'affirment de plus en plus, il y a des structures qui restent excessivement régionales. Le parallélisme est ici intéressant, mais il ne faudrait pas tomber dans le déterminisme en disant que c'est parce qu'il y a l'un que l'autre apparaîtra nécessairement.

\section{NSQV - Vous auriez un exemple concret à présenter ?}

JMC - Depuis qu'il a opéré un rassemblement territorial autour de sa personne, le duc Philippe le Bon dispose d'un Conseil - qui n'a d'ailleurs aucun autre nom spécifique - dans lequel siègent des personnes qui ne représentent pas formellement ses différents territoires même s'ils en sont issus. Il faut ensuite attendre la fin des années 1440 et le début des années 1450 pour rencontrer un texte qui prétende organiser un conseil. La démarche institutionnelle précède ici le texte. Plus tard, Charles le Hardi, dit le Téméraire, qui a en tête un véritable projet politique, rédige des textes et c'est par leur biais que les choses se mettent en place. La chronologie est ici inversée par rapport à ce que l'on pouvait observer durant le gouvernement de son père. Il n'y a donc rien d'absolu, mais sans aucun doute une imbrication.

NSQV - Pour le $15^{\mathrm{e}}$ siècle vous mettez souvent en avant qu'on ne dispose presque pas de documents préparatoires. A contrario, pour le $16^{\mathbf{e}}$ siècle et singulièrement à partir de Philippe II, cette observation est différente. Des projets entiers sont conservés avec les annotations, par exemple. Comment expliquer cette évolution ? Une conjonction entre le facteur institutionnel (nouvelles structures qui se mettent en place) et les balbutiements d'une vision archivistique sous Charles Quint puis Philippe II ? Voyez-vous d'autres facteurs?

JMC - Ici la réponse est dans la question. Je crois qu'au $16^{\mathrm{e}}$ siècle il s'agit d'une autre organisation sur le plan archivistique. Il est possible et même probable que l'on produisait plus parce que le rapport à l'écrit était peut-être autre. Selon moi, dire qu'on a mieux conservé les archives du $16^{\mathrm{e}}$ siècle que celles du $15^{\mathrm{e}}$ siècle n'est pas déterminant. Les occasions de destruction ont été aussi nombreuses. Après tout, le bombardement de Bruxelles en 1695 a détruit autant de documents du $16^{\mathrm{e}}$ et du $17^{\mathrm{e}}$ que du $14^{\mathrm{e}}$ et du $15^{\mathrm{e}}$ siècle. Je crois donc que ce sont bien les habitudes et les usages ainsi que l'organisation de services archivistiques qui sont déterminants. Prenons la Chambre des comptes. Grâce aux travaux de Jean-Baptiste Santamaria26 et, pour une époque postérieure, celui plus modeste de Mireille Jean27, on constate qu'il y a une organisation des services. On ne se contente plus de dire qu'on a produit des comptes, des quittances, etc. Il s'agit d'organiser toute cette documentation. Cette organisation ne peut que produire des fruits et par conséquent je crois qu'une vision archivistique, même à ses balbutiements, est un élément d'explication déterminant. Beaucoup plus en tout cas que des facteurs de conservation. On pouvait peut-être détruire plus facilement puisqu'on n'était pas organisé pour conserver ce qu'on produisait.

NSQV - Depuis plusieurs décennies les historiens tentent d'aborder la question de l'État moderne et de sa genèse via l'impact des sujets (approche bottom-up) et ce dans une perspective inverse à celle qui a longtemps prédominé (l'Etat est une construction des seuls gouvernants, approche top-down). On en retrouve d'ailleurs quelques mentions dans 
Une vie dédiée à Clio sous le prisme de la loi, du Hainaut et des ducs de Bou...

votre ouvrage écrit avec Hugo De Schepper28. Or, depuis quelques années on trouve une approche un peu intermédiaire qui tend à dire que les relations entre gouvernants et gouvernés pouvaient être émancipatrices pour les deux parties (l'empowerment29). Quel est votre avis là-dessus ? Où placeriez-vous le «curseur »? En lien avec cette question on pourrait rappeler un autre débat lancinant : dans quelle proportion les princes et souverains ont-ils légiféré motu proprio ou sur requête ?

JMC - Je vais commencer par la fin en disant que je crois de moins en moins au poids du motu proprio. Je suis de plus en plus persuadé qu'il y a toujours une impulsion qui est donnée. Autrefois certains historiens du droit et des institutions semblaient hésiter à le dire parce que ça leur aurait peut-être donné mauvaise conscience, comme s’ils diminuaient le pouvoir du prince...

\section{NSQV - Comme si, au final, le pouvoir princier aurait été comme une coquille vide ?}

JMC - Oui, c'est ça. Le motu proprio n'occupant que peu de place, les historiens étaient presque en train de culpabiliser. Je pars plutôt de l'idée - c'est sans doute une conviction personnelle - que le « bon prince », pour autant qu'il existe, c'est celui qui affirme qu'il est le prince, qui dit « le prince, c'est moi ». C'est donc celui qui décide, mais qui avant de le faire écoute. J'utilisais, par exemple, dans mes cours d'histoire du droit et d'histoire des temps modernes, cette remarque - j'ai oublié de qui elle était - à propos de Louis XIV : il décidait seul mais non en solitaire. La première fois où j'ai lu cette formule, j’y ai beaucoup réfléchi. Certains historiens qui s'attachent aux princes qu'ils étudient - on s'attache tous à des figures, moi y compris - peuvent parfois renâcler à dire qu'ils étaient autoritaires. Alors on dit que le prince prenait conseil. C'est très bien de dire qu'il prenait conseil, mais au moment de décider le « bon prince » est celui qui affirme qu'il est le seul à décider. Certes, le prince peut avoir des idées, mais le plus souvent je pense qu'il y a quelque chose derrière. Ça peut venir de son bras droit, de son bras gauche, de son petit orteil, bref ça peut venir de très loin. La vraie question n'est donc pas de se demander s'il y a beaucoup d'actes pris motu proprio. Elle consiste plutôt à se demander dans quelle mesure et pourquoi mentionne-t-on ici et pas là-bas l'impulsion qui a été donnée. J'utilise le mot impulsion pour rester volontairement neutre car si je parle de « requête », cela donne déjà des orientations.

NSQV - Parce que ça peut être un rapport d'officier, un mémoire d'un conseil provincial, etc.

JMC - Exactement. Quand Georges Chastellain dit de Nicolas Rolin, chancelier de Philippe le Bon, qu'il était le regardeur sur tout, ça veut tout dire ! Nicolas Rolin était celui qui disait au duc ce qu'il devait faire et on sait, par ailleurs, la grande confiance qu'avait Philippe le Bon qui était un piètre politique pour son chancelier. Évidemment Philippe le Bon n'allait pas clamer que son chancelier lui avait dit de faire ceci ou cela. D’ailleurs, Nicolas Rolin n'avait pas besoin de ça pour être mis en évidence. Chastellain n'écrivant pas pour ne rien dire, c'est d'autant plus fort qu'il présente Rolin comme le regardeur sur tout. C'est tout de même très fort quand on y pense ! Interprété négativement - quoique je sois porté à m’en garder -, cela pourrait signifier que Rolin était le mêle-tout, celui qui voulait être calife à la place du calife.

NSQV - Le « vrai » motu proprio est une illusion si on vous suit bien.

JMC - Un prince peut avoir une idée voire une lubie, mais je pense qu'il y aura souvent quelque chose derrière. C'est alors passionnant d'essayer de trouver à quoi on fait référence. On peut 
mentionner une requête dans le cas, par exemple, où on veut se concilier une ville. On retrouve des expressions du type «Nos bons sujets nous ont dit que... » et tous ces poncifs que l'on retrouve à des moments différents de l'acte. Il y en a d'ailleurs un qui me plait toujours qui est plus ou moins comme ceci : « ils nous ont fait valoir que leur ville est petite, n'ayant rivière portant bateau... ». Pour le coup, il s'agit vraiment d'une expression... bateau que l'on retrouve à plusieurs reprises. Ça veut bien dire qu'il y a eu une démarche préalable et on a cherché une formule pour en rendre compte. Parfois on peut également râler devant des mentions très générales du type « ayant eu bon avis et mûr conseil » ou «nous avons été informé ». Le vrai motu proprio devait donc être quelque chose de relativement limité.

\section{NSQV - Si on en revenait à la première partie de notre question concernant les approches qui ont pu être envisagées pour caractériser la genèse de l’Etat moderne.}

JMC - Je me rallierais à cette approche intermédiaire (empowering interactions) qui tente de concilier les points de vue. C'est d'ailleurs aussi symptomatique par rapport à mes propres recherches où j'en suis arrivé à m'intéresser plus directement aux assemblées représentatives même si elles n’avaient jamais été absentes de mon horizon. L'article dans les Mélanges Philippe Guignet sur les destinées des États provinciaux aux temps modernes pouvait peut-être paraître surprenant à première vue $\underline{30}$. Mais je crois de plus en plus qu'il faut aller voir ce qui se passe à la base, du côté des États. Inutile de dire que ce n'est pas la lecture de la thèse de Marie Van Eeckenrode qui allait me distraire de ce dessein. Elle montre très bien qu'avec les États il y a quelque chose qui bout et fourmille et ce dans une principauté, le Hainaut, où ça ne part pas dans tous les sens.

\section{NSQV - La relation gouvernant-gouvernés doit-elle alors être vue en termes de litige ?}

JMC - On ne doit pas nécessairement voir cette relation sous la forme d'un litige. Ce n'est pas un combat pour savoir qui, de l'un ou de l'autre, va l'emporter. C'est davantage s'interroger sur l'endroit où ils parviennent vraiment à se rencontrer. On a peut-être tendance à mettre fort en avant que les grands moments des assemblées sont ceux où elles tapent du poing sur la table (Grand Privilège de 1477, Pacification de Gand en 1576). Les États font certes beaucoup de bruit à ces moments, mais pourquoi considérer que ce qui est plus important dans l'histoire d'une institution ou d'un règne c'est le moment où l'on se bagarre ? Les travaux de Violet Soen pour le $16^{\mathrm{e}}$ siècle sont à cet égard vraiment remarquables $\underline{31}$.

\section{NSQV - Pour quelle raison?}

JMC - Je caricature peut-être un peu, mais plutôt que de voir ce qui divise entre gouvernants et gouvernés, tentons de voir ce par quoi ils se sont rapprochés. C'est ce que Violet Soen abordait dans son ouvrage sur les pardons. Même dans son dernier livre, elle tente de voir sur quoi on se met d'accord. Elle ne privilégie pas seulement les causes de chamailleries. On constate ainsi que tant un duc d'Albe qu'un Granvelle - qui ne sont tout de même pas des doux - ont tendance à dire à Philippe II, à Madrid, qu'il devrait voir sur quel terrain il pourrait négocier et traiter avec les Pays-Bas. Il y a l'idée de diviser pour régner, de traiter avec Pierre pour écarter Paul. C'est tout de même assez neuf de mettre l'accent là-dessus. De même, quand on fait l'histoire de la législation il ne faut sans doute pas voir la loi comme une confrontation et imposition mais plutôt comme une rencontre. Je prône volontiers cette approche et je crois que je ne me situerais pas trop mal dans cette perspective de l'empowerment. 
Une vie dédiée à Clio sous le prisme de la loi, du Hainaut et des ducs de Bou...

\section{Notes}

1 Cauchies J.-M., La législation princière pour le comté de Hainaut. Ducs de Bourgogne et premiers Habsbourg (1427-1506) - Contribution à l'étude des rapports entre gouvernants et gouvernés dans les Pays-Bas à l'aube des temps modernes, Bruxelles, Presses de l'Université Saint-Louis - Bruxelles, 1982, 670 p.

$\underline{2}$ Les Facultés universitaires Saint-Louis d'alors ne disposaient pas encore de l'autorisation pour délivrer des diplômes de troisième cycle (doctorat) comme c'est aujourd'hui le cas.

$\underline{3}$ Juriste et historien de formation, Philippe Godding est l'auteur d'une thèse de doctorat consacrée au droit foncier à Bruxelles au moyen âge et d'un ouvrage majeur dédié au droit privé dans les Pays-Bas méridionaux $\left(12^{\mathrm{e}}-18^{\mathrm{e}}\right.$ siècle). Il fut par ailleurs professeur aux Facultés universitaires Saint-Louis et à l'Université catholique de Louvain. Il quitta ses fonctions aux Facultés Saint-Louis lorsqu'il devint doyen de la faculté de Droit à l’Université catholique de Louvain (Louvain-la-Neuve).

$\underline{4}$ En tant que correspondant. Nommé membre titulaire le 15 mai 2004.

$\underline{5}$ CAuchies J.-M., De geschiedenis van de wetgeving (Middeleeuwen - Moderne Tijden) : een mijnenveld ?, dans Uuytwysens d'Archiven. Handelingen van de XIe Belgisch-Nederlandse rechtshistorische dagen, Louvain, 1992, p. 1-26.

6 EBEL F., Geschichte der Gesetzgebung in Deutschland, Göttingen, 1988.

7 En référence aux quelques 350 principautés composant l'ancien Saint-Empire germanique. Jean-Marie Cauchies met en avant des recherches sur la Bavière, la Hesse, etc., où le pouvoir d'un prince territorial, parfois puissant, s'exerce sur un territoire donné. Il n’y a pas besoin d'avoir un pouvoir qui serait celui d'une «Allemagne » et pourtant il y a des princes-législateurs.

8 Jean-Marie Cauchies entend rédiger un ouvrage de synthèse sur la loi dans les Pays-Bas méridionaux du $12^{\mathrm{e}}$ au $18^{\mathrm{e}}$ siècle. Cet ouvrage devra paraître, à terme, à l'Académie royale de Belgique.

9 CAUChIES J-M, Le processus de la décision politique à travers quelques actes des ducs de Bourgogne (1429-1472), dans Publications du Centre européen d'études bourguignonnes, 1984, t. 14, p. 42

10 Né le 24 mars 1914, décédé le 10 juin 2001. Historien belge originaire du Hainaut, spécialiste du moyen âge. Professeur à l’Université libre de Bruxelles (ULB).

11 ARnould M., Les dénombrements de foyers dans le comté de Hainaut (XIV $-\mathrm{XVI}^{\mathrm{e}}$ siècle), Bruxelles, Palais des Académies, 1956, XXIV-771 p.

12 Voir SCHNERB B., Louis XI, roi chasseur, dans Bien dire et bien aprandre. Revue de médiévistique, t. 27, 2009, p. 69-84.

13 CaUchies Jean-Marie, La législation de Philippe le Bon, duc de Bourgogne, dans les Pays-Bas autour de 1430 : des ambitions réformatrices ?, communication au colloque international La loi du Prince. Les Statuta Sabaudiae d’Amédée VIII, Université de Genève, 2-4 février 2014. 
14 JouAnNA A., Le pouvoir absolu : naissance de l'imaginaire politique de la royauté, Paris, Gallimard, 2013, p. 130.

15 CAuchies J.-M., La Constitution, le serment et le prince dans le Hainaut ancien, dans Liber amicorum John Gilissen. Code et constitution : mélanges historiques, Anvers, 1983, p. 51-60.

16 Professeur émérite d’histoire médiévale à l’UGent, ancien président de la Commission royale d'histoire.

17 Thèse publiée quatre ans plus tard : NAZET J., Les chapitres de chanoines séculiers en Hainaut du XII ${ }^{\mathrm{e}}$ au début du XV $\mathrm{X}^{\mathrm{e}}$ siècle, Bruxelles, Académie royale de Belgique, 1993.

18 Professeur d’histoire médiévale à l’Université catholique de Louvain (Louvain-la-Neuve).

19 VAN EECKENRODE M., Les États de Hainaut : servir le prince, représenter le pays : une assemblée dans les allées du pouvoir (ca. 1400-1550), Louvain-la-Neuve, Université catholique de Louvain, 2015 (thèse inédite en histoire). Voir également ID., Les États de Hainaut sous le règne de Philippe le Bon 1427-1467, Courtrai, UGA, 2011.

20 CAUChies J.-M., Le « cri » et l'espace urbain : bretèches et publication dans les villes des anciens Pays-Bas, dans Villes et villages. Organisation de l'espace. Mélanges offerts à Jean-Marie Duvosquel..., Bruxelles, 2011, p. 167-189 (Revue belge de philologie et d’histoire, t. 89, 2011).

21 WijfFels A., Qui millies allegatur. Les allégations du droit savant dans les dossiers du Grand Conseil de Malines (causes septentrionales, ca. 1460-1580), Leyde, 1985, XLII-1173 p. (Rechtshistorische Studies, t. XI).

22 De Schepper H., De Grote Raad van Mechelen, hoogste rechtscollege in de Nederlanden ?, dans Bijdragen en Mededelingen betreffende de Geschiedenis der Nederlanden, 1978, t. 93, p. 358-376.

23 Ost F., Le temps du Droit, Paris, 1999, p. 224-225.

24 CAuchies J.-M., « Oyez et faites paix... » De Baudouin de Constantinople à Joseph II : six siècles de législation aux anciens Pays-Bas, dans Académie royale de Belgique. Bulletin de la Classe des lettres et des sciences morales et politiques, 6 ${ }^{\mathrm{e}}$ s. t. XIV, 2003, p. 287-309.

25 SimON N., La législation de Charles Quint dans les Pays-Bas : jeux et enjeux de contorsions identitaires, dans L'identité au pluriel. Jeux et enjeux des appartenances autour des anciens PaysBas, XIV ${ }^{\mathrm{e}}$-XVIII ${ }^{\mathrm{e}}$ siècles / Identity and Identities. Issues of Belonging in the Low Countries, $14^{\text {th }}-18^{\text {th }}$ centuries, (dir.) Y. JunOt, F. MARIAGE et V. Soen, Villeneuve d'Ascq, 2014, p. 41-58 (Revue du Nord, Hors-série, collection Histoire, $\left.n^{\circ} 30\right)$.

26 SANTAMARIA J.-B., La Chambre des comptes de Lille (1386-1419). Essor, organisation et fonctionnement d’une institution princière, Turnhout, Brepols, 2012.

27 JEAN M., La Chambre des comptes de Lille, l'institution et les hommes (1477-1667), Paris, École des chartes, 1992.

28 CAuchies J.-M. et DE Schepper H., Justice, grâce et législation. Genèse de l’État et moyens 
Une vie dédiée à Clio sous le prisme de la loi, du Hainaut et des ducs de Bou...

juridiques dans les Pays-Bas, 1200-1600, Bruxelles, 1994, 127 p. (F.U.S.L. Centre de recherches en histoire du droit et des institutions. Cahiers, 2).

29 Blockmans W., Holenstein A. et Mathieu J. (dir.) (avec la collab. de D. SchläPPI), Empowering Interactions. Political Cultures and the Emergence of the State in Europe 1300-1900, Farnham, Ashgate, 2009.

30 CAuchIes J.-M., Les assemblées d'états provinciaux aux anciens Pays-Bas (XVI ${ }^{\mathrm{e}}$-XVIII ${ }^{\mathrm{e}}$ siècles) : apogée, soubresauts ou déclin ?, dans La ville à l'époque moderne. Mélanges en l'honneur de Philippe Guignet, textes réunis par M.-L. LEGAY, Villeneuve-d’Ascq, 2013, p. 669-679 (Revue du Nord, t. 95, nos 400-401).

31 Professeure d'histoire moderne à la KU Leuven. Parmi ses travaux retenons : SoEN V., Geen pardon zonder paus! Studie over de complementariteit van het koninklijk en pauselijk generaal pardon (1570-1574) en over inquisiteur-generaal Michael Baius (1560-1576), Bruxelles, 2007 (Verhandelingen van de Koninklijke Vlaamse Academie van België voor Wetenschappen en Kunsten, nieuwe reeks 14) ; ID., Vredehandel. Adellijke en Habsburgse verzoeningspogingen tijdens de Nederlandse Opstand, Amsterdam, 2012.

PDF généré automatiquement le 2020-07-08 07:01:57

Url de l'article : https://popups.uliege.be:443/1370-2262/index.php?id=346 